\title{
Medical devices for the anesthetist: current perspectives
}

This article was published in the following Dove Press journal:

Medical Devices: Evidence and Research

25 March 2014

Number of times this article has been viewed

Jerry Ingrande

Hendrikus JM Lemmens

Department of Anesthesiology, Perioperative and Pain Medicine, Stanford University School of Medicine, Stanford, CA, USA
Correspondence: Jerry Ingrande Department of Anesthesia, Perioperative and Pain Medicine, Stanford University School of Medicine, 300 Pasteur Drive, Room H3580, Stanford, CA, USA

Tel + I 6507237377

Fax +I 6507258544

Email jerryi@stanford.edu
Abstract: Anesthesiologists are unique among most physicians in that they routinely use technology and medical devices to carry out their daily activities. Recently, there have been significant advances in medical technology. These advances have increased the number and utility of medical devices available to the anesthesiologist. There is little doubt that these new tools have improved the practice of anesthesia. Monitoring has become more comprehensive and less invasive, airway management has become easier, and placement of central venous catheters and regional nerve blockade has become faster and safer. This review focuses on key medical devices such as cardiovascular monitors, airway equipment, neuromonitoring tools, ultrasound, and target controlled drug delivery software and hardware. This review demonstrates how advances in these areas have improved the safety and efficacy of anesthesia and facilitate its administration. When applicable, indications and contraindications to the use of these novel devices will be explored as well as the controversies surrounding their use.

Keywords: catheters, echocardiography, ultrasound, fiberoptic bronchoscope, laryngeal mask airway, closed-loop anesthesia

\section{Introduction}

Perhaps no other medical specialty has benefited more from recent technological advances as the specialty of anesthesiology. Use of sophisticated medical devices and technology is now commonplace in the field of anesthesia, and because they are used on a daily, case-by-case basis, they can be overlooked and taken for granted. In the last 2 decades, technology associated with the specialty of anesthesia has evolved a great deal. Advances in fiberoptic technology have allowed the development of video laryngoscopy, simplifying the approach to management of a patient's airway. Increases in computational power and novel mathematical modeling techniques have led to the development of target controlled infusion devices and software, changing how drugs are administered. New monitoring devices such as echocardiography and ultrasound (US) have now become mainstream. New technology has made the practice of anesthesia more efficient and, most importantly, has improved patient safety. The following is a review of the most pertinent technological advances in the specialty of anesthesia and their influence on the field.

\section{Cardiovascular devices}

Intravascular catheters: central venous and pulmonary arterial catheters

Central venous catheters (CVCs) are commonly employed for intravascular access and for assessment of intravascular volume. CVCs are used for rapid administration 
of fluids, treatment of venous air emboli, and assessment of central venous pressure. The benefit of central venous pressure monitoring is not without debate. Use of a CVC is the most common measurement of static preload. ${ }^{1}$ It can provide an accurate measurement of right ventricular filling pressures and preload. Central venous pressures are not as reliable for assessment of left-sided pressures, especially in the presence of cardiopulmonary disease. The use of CVCs for assessment of fluid responsiveness has recently been scrutinized. A recent study comparing pulmonary capillary wedge pressures with central venous pressure demonstrated that central venous pressure did not significantly predict fluid responsiveness. ${ }^{2}$ Forrester et $\mathrm{al}^{3}$ showed that central venous pressures did not correlate with the presence or absence of pulmonary congestion in patients with acute myocardial infarction. Furthermore, central venous pressures were not an accurate indicator of directional changes in pulmonary capillary wedge pressures during fluid administration. ${ }^{3}$

Placement of a pulmonary artery catheter (PAC) through a CVC provides a more accurate assessment of left-sided heart pressures and may be a valuable tool for the perioperative assessment of cardiovascular function. It has been estimated that $55 \%$ of the 1.5 billion PACs inserted annually in the United States are placed in high-risk surgical and trauma patients. ${ }^{4}$ A PAC can measure left-sided filling pressure, cardiac output, and mixed venous oxygen saturation. Proponents of the PAC advocate their use because this additional information may help evaluate fluid and drug responsiveness in critically ill surgical patients. In fact, studies that evaluated the utility of PACs in surgical and critically ill patients have reported that information gained from their use contributed to changes in management in oneto two-thirds of all cases..$^{5-9}$ Studies examining the clinical outcomes in surgical and critically ill subjects receiving PACs have yielded conflicting results, fueling debate about the utility of PACs. Wilson et $\mathrm{al}^{10}$ randomized 138 high-risk surgical patients undergoing elective surgery to determine whether preoperative optimization of oxygen delivery affected outcome. Intervention groups received PACs plus ionotropes, whereas control subjects received "routine care" (no invasive monitors or ionotropes). Subjects in the treatment groups who received fluids and ionotropes guided by PACs had lower mortality and decreased length of hospital stay. The investigators concluded that fluid optimization was the major contributor to improved oxygen delivery, and that noninvasive monitoring may leave patients volume depleted. Two studies have demonstrated that trauma subjects given goal-directed care based upon data obtained from a PAC (cardiac index, oxygen consumption, and oxygen delivery) had improved survival and decreased end-organ failure compared with control subjects. ${ }^{11,12}$

Detractors of PAC use argue that these devices contribute to no changes in patient outcome, or even increase morbidity. In a large, multicenter, prospective cohort study of 5,735 propensity-matched subjects, Connors et al ${ }^{13}$ found that critically ill subjects who received a PAC within 24 hours of admission had a significantly higher 30-day mortality and increased use of resources compared with subjects who did not receive a PAC. However, the authors could not comment on the source of lack of benefit of the PAC. In addition, a prospective study of 120 subjects randomized to intervention (PAC) or no intervention found that subjects who received a PAC prior to surgery received more fluid in the pre- and postoperative period and had an increased number of adverse events postoperatively. ${ }^{14}$ In a direct comparison of PACs with CVCs in low-risk subjects undergoing coronary artery bypass grafting, Stewart et $\mathrm{al}^{15}$ found that subjects who received a PAC had longer intubation times compared with subjects with a CVC. There were no significant differences in intensive care unit length of stay. However, in a retrospective cohort analysis of subjects undergoing nonemergent coronary artery bypass grafting, PACs were associated with an increased risk of inhospital mortality, greater length of stay, and higher costs. ${ }^{16}$

The conflicting reports of the clinical effectiveness of PACs and the limitations of the evidence have spurred the American Society of Anesthesiologists (ASA) to recommend practice guidelines for the use of PACs. ${ }^{17,18}$ Ultimately, the task force recommended that PAC placement is appropriate in the setting of surgical procedures associated with hemodynamic complications or in patients entering surgery with pre-existing risk factors for hemodynamic perturbations. In addition, the level of risk must be assessed in the setting of physician skills in both placement and interpretation of the data and in technical support related to the PAC.

\section{Transesophageal echocardiography}

The use of transesophageal echocardiography (TEE) in the perioperative period for direct, real-time evaluation of cardiac function is expanding. ${ }^{19} \mathrm{TEE}$ is used extensively for cardiac surgery for diagnostic purposes and for hemodynamic monitoring. TEE is used for assessment of cardiac output, left ventricular filling, and valvular, atrial, and ventricular function. ${ }^{20}$ During cardiac surgery, TEE has been shown to be an influential monitoring device guiding perioperative decision making. Bergquist et $\mathrm{al}^{21}$ demonstrated that TEE was the most important factor guiding decision making in nearly $20 \%$ of 587 cardiac procedures assessed. In a prospective study analyzing the utility of TEE in cardiac surgery, Mishra et $\mathrm{a}^{22}$ 
found that out of over 5,000 cases, 36\% had TEE-guided hemodynamic interventions, and in $23 \%$ of cases, TEE was the sole guiding factor. A similar study demonstrated that TEE changed medical management in $53 \%$ of cases and changed surgical management in $30 \%{ }^{23}$

TEE has proven to be an important tool for the management of pediatric congenital heart disease. TEE has been shown to aid in both the diagnosis of congenital heart disease and the operative management of patients with congenital heart disease. Guzeltas et $\mathrm{al}^{24}$ performed a retrospective analysis of 1,008 pediatric patients undergoing surgery for congenital heart disease between 2009 and 2013. Of the 265 patients in whom TEE was used, preoperative diagnosis was confirmed in 260 patients. In the remaining five patients, surgical intervention was changed following TEE. In addition, in twelve patients, cardiopulmonary bypass was reinitiated following repair for residual defects identified by TEE. The authors concluded that intraoperative TEE should be standard during anesthesia for repair of pediatric congenital heart disease.

In a similar study of 865 pediatric patients undergoing surgical repair of congenital heart disease, TEE was responsible for surgical alterations in $12.7 \%$ of cases and reinstitution of cardiopulmonary bypass in $7.3 \%$ of cases. ${ }^{25}$ For its role in diagnosis of congenital heart disease and for evaluation of repair, TEE has been shown to be an important and costeffective monitor for pediatric congenital heart surgery. ${ }^{26}$ Complications associated with its use have been shown to be minimal and without long-term sequelae. ${ }^{26}$

TEE is a popular and instrumental monitoring device for cardiac surgery. Its use in noncardiac surgery is not as widespread but is equally promising. TEE can be an instrumental tool in guiding decision making in complex noncardiac cases such as liver transplantation, complex abdominal surgery, trauma, and critically ill patients. TEE has been shown to be an important tool for assessment of hemodynamic function during liver transplantation. ${ }^{27}$ Hemodynamic instability is a frequent occurrence during liver transplantation, usually due to blood loss or surgical manipulation. The use of TEE during these procedures allows discrimination of the etiology of hemodynamic instability by allowing visualization of cardiac filling (preload) and cardiac function (pump failure). In a large study examining the use of TEE in high-volume liver transplantation centers, TEE is used by $86 \%$ of anesthesiologists during liver transplantation cases, although only $12 \%$ of these were board certified in the use of TEE. ${ }^{28}$

\section{Noninvasive cardiac output monitors}

Continuous cardiac output (CCO) monitoring allows the anesthesiologist the opportunity to react to acute changes in hemodynamic and ventricular performance in real time. TEE and pulmonary arterial catheters are two common methods by which $\mathrm{CCO}$ is monitored perioperatively. However, these two devices involve invasive maneuvers and require skill and prior training in their use and interpretation. New devices have recently been developed that allow $\mathrm{CCO}$ measurements to be carried out noninvasively or with the use of a pre-existing arterial line catheter. These devices use arterial waveform analysis to measure $\mathrm{CCO}$ and stroke volume variation (SVV) or pulse pressure variation (PPV). A mathematical algorithm is used to calculate $\mathrm{CCO}$ from the arterial waveform analysis. Cardiac output is determined by calculating the area under the curve of the systolic portion of the arterial waveform divided by the aortic impedance multiplied by the heart rate. ${ }^{29}$ As arterial line catheters are usually inserted in high-risk patient populations, this technology is likely to be readily available to these patients as well as in awake patients in whom use of TEE or PAC is technically difficult. Both SVV and PPV have been shown to accurately predict fluid responsiveness in subjects undergoing surgery. ${ }^{30,31}$ However, a recent study has shown that both SVV and PPV offer little predictive value in fluid responsiveness during surgery. ${ }^{32}$ Furthermore, when compared with Doppler echocardiography, which is considered the gold standard for cardiac output measurement, noninvasive methods for measuring SVV and PPV show weak correlation and low sensitivity and specificity. ${ }^{32,33}$ However, when compared with thermodilution-based cardiac output measurements, noninvasive cardiac output techniques have showed good correlation and small bias. ${ }^{34,35}$ Considering the equivocal nature of the utility of SVV and PPV in the perioperative setting, results must be taken into clinical context and interpreted with caution. Nevertheless, when echocardiography or the use of PACs is contraindicated, these noninvasive devices may be considered for clinical guidance.

\section{Airway devices Fiberoptic bronchoscope}

The fiberoptic bronchoscope (FOB) has become a ubiquitous and important tool for airway management since its first reported use in $1967 .{ }^{36}$ Unlike other airway devices, the FOB is unique because its length, flexibility, and optics allow direct visualization of anatomic structures, including the vocal cords, while allowing direct passage through the vocal cords. For these reasons, the FOB can be used not only to aid tracheal intubation but also for confirming placement of an endotracheal tube or to aid in the diagnosis of tracheal or bronchial pathology.

Although there are no true indications for its use, the FOB is a valuable tool for airway management in anticipated and unanticipated difficult intubation, in situations where 
cervical manipulation may be contraindicated, or in cases of upper and lower airway obstruction. ${ }^{37}$ With the use of adequate airway topicalization with local anesthetics, the FOB may be used in an awake, spontaneously ventilating patient, especially in cases where an anticipated difficult airway exists. ${ }^{38}$ ASA practice guidelines for management of difficult airways indicate the use of awake intubation as the gold standard approach. ${ }^{39}$ The use of the FOB has been described as the most useful aid for awake intubation in a patient with a known difficult airway. ${ }^{40}$

The contraindications to the use of the FOB are relative but merit consideration. Use of the FOB requires fine psychomotor skills to navigate the scope through the pharynx into the trachea. Heavy airway secretions or bleeding may limit the view through the scope. Antisialagogues such as glycopyrrolate or atropine may be used to limit secretions; however, care must be taken to ensure that the patient can tolerate increases in heart rate. The patient must be cooperative and still if an awake approach is planned. In addition, in the setting of hypoxia or impending hypoxia, the time needed to advance the FOB through the vocal cords may limit the utility of the FOB, especially if concurrent ventilation is not used. In fact, analysis of the ASA closed claims database reveals that death or brain damage occurred in $75 \%$ of twelve reported cases in which awake intubation was used for an anticipated difficult airway. ${ }^{41}$ The use of sedation, bleeding, technical factors, and lack of patient cooperation were listed as reasons for failure.

The FOB is a useful device for rescue and planned intubation. Nearly all surveyed members of the ASA claim to have the FOB available to them. ${ }^{42}$ However, use of the FOB requires training and skill. Due to the advanced skills necessary to use the FOB, experts have stated that all trained anesthesiologists should become familiar with its use. ${ }^{43}$

\section{Video laryngoscopes}

Video laryngoscopes, like direct laryngoscopes, consist of a handle and blade and are inserted into the pharynx in a similar manner. However, video laryngoscopes are equipped with a video camera on the distal end of the blade, allowing the laryngoscopist an indirect view of the vocal cords via an attached screen. Various video laryngoscopes are widely available but often mimic conventional Macintosh-type blades or angulated blades, or consist of a curved-type blade with an attached channel to pass an unstyletted endotracheal tube.

Because of their resemblance to conventional laryngoscopes, the skills necessary to use video laryngoscopes are, in general, easy to master, and the devices are associated with high success rates. In a study comparing success of laryngo- scopy by novices, novices trained with video laryngoscopes had better success rates compared with novices trained with conventional laryngoscopes. ${ }^{44}$ In a study of 235 intubations, experienced anesthesiologists had a 99.6\% success rate at intubation when using a video laryngoscope without any prior preparation or experience. ${ }^{45}$ Due to the relative ease of use and success rate of video laryngoscopes, some authors have championed the use of video laryngoscopes as valid rescue devices for tracheal intubation after failure of direct laryngoscopy. ${ }^{46,47}$ A recent study comparing video laryngoscopy with FOB-aided awake intubation in patients judged to be potentially difficult to intubate found no difference in the times to achieve tracheal intubation between the two methods. ${ }^{48}$ The authors concluded that video laryngoscopy could be a viable alternative to FOB-aided intubation. However, this study used an invasive, transtracheal injection of lidocaine to achieve airway topicalization in both groups. The adequacy of video laryngoscopy for awake intubation using noninvasive airway topicalization has been questioned. ${ }^{49}$

\section{Laryngeal mask airway}

The laryngeal mask airway (LMA) is a supraglottic device that is designed to sit in the posterior pharyngeal wall, providing an aperture above the trachea with no direct contact with the tracheal mucosa. A cuff allows for a relatively tight seal with little leakage of gas during spontaneous ventilation. However, despite optimal placement of the LMA and inflation of the cuff, the seal is not as robust as that of an endotracheal tube.

The inventor of the prototype LMA, Dr Archie Brain, ${ }^{50}$ created the LMA as a device able to offer the advantages of endotracheal intubation, while avoiding the requirement of vocal cord visualization and abduction via a tube. In a small, preliminary study, the LMA was used successfully in $99 \%$ of cases. ${ }^{50}$ Proponents of its use claim that by sitting above the vocal cords, the incidences of laryngospasm, sore throat, and coughing are minimized compared with the endotracheal tube ${ }^{51}$ In addition, insertion of the LMA may be associated with a more favorable hemodynamic response, with less hypertension and tachycardia than direct laryngoscopy and endotracheal intubation.

One of the major drawbacks of the LMA is that it does not pass through the vocal cords. Because of this, the LMA does not provide a definitive airway and may not protect the airway from secretions and aspiration as well as an endotracheal tube. Keller et $\mathrm{al}^{52}$ describe a series of aspirations in subjects in whom an LMA was used. In addition, the same authors described an additional 20 published cases of aspiration associated with LMA use. The authors state that in all 
but one case there were pre-existing risk factors for aspiration that may have contraindicated the use of the LMA. ${ }^{52}$ However, although an LMA may not be effective in preventing gastric aspiration, it may be protective against aspiration of supraglottic secretions and blood. In a case series of 217 ear, nose, and throat cases in which an LMA was used, Daum and O'Reilly ${ }^{53}$ described how blood and airway secretions safely pooled above the device with no contamination of the airway reported. Nevertheless, because the LMA does not definitively protect against aspiration, its use should be questioned in cases and subjects who are at increased risk. These include decreased gastric emptying (diabetes, chronic opioids, gastroparesis), increased intra-abdominal pressure (lithotomy, laparoscopy, pregnancy, obesity), and in subjects with full stomachs (trauma, emergency surgery). Studies comparing risks associated with LMA use compared with endotracheal intubation are generally limited to retrospective analyses. In addition, because the incidence of aspiration is very small, prospective studies analyzing the safety of the LMA compared with endotracheal intubation are difficult to conduct and are therefore lacking.

\section{Neuromonitoring Electroencephalogram}

Monitoring the electroencephalogram (EEG) during anesthesia is an important tool for the assessment of cerebral function. Intraoperative EEG monitoring is used primarily during neurosurgery and cardiac surgery to detect cerebral ischemia or epileptic foci and for the assessment of pharmacologic interventions. Interpretation of EEG changes can help guide neurosurgical intervention and intraoperative decision making.

EEG monitoring uses scalp electrodes to monitor large areas of the cerebral cortex, consisting of superficial pyramidal cells. ${ }^{54}$ The pyramidal cells are sensitive to hypoxia and ischemia, and decreases in cerebral blood flow (as can occur during neurovascular surgery) can be detected by changes in EEG activity. ${ }^{55}$ Changes in EEG typically occur between 30 seconds and 5 minutes following ischemic changes. ${ }^{55}$

Use of EEG monitoring during carotid endarterectomy is widespread, as changes in the EEG waveform can guide intraoperative decision making. Rapid flattening of the EEG not responsive to increases in blood pressure after clamping of the carotid artery indicates that clamping is not being tolerated and shunt placement is indicated. Intraoperative changes in the EEG waveform, however, are associated with a high number of false positive results. Nevertheless, EEG monitoring has been shown to reduce ischemic time and risk of stroke during carotid endarterectomy. ${ }^{56}$ A study analyzing
658 subjects undergoing carotid endarterectomy with EEG monitoring showed that 34 subjects had a postoperative neurologic deficit. ${ }^{57}$ Only seven of these were due to cerebral hypoperfusion. ${ }^{57}$

Analysis of EEG has gained popularity not only in neurovascular cases but also in patients and cases where there is an increased risk for intraoperative awareness. Generally, when a potent inhalational anesthetic agent is used for hypnosis, monitoring end-tidal anesthetic concentration and titration to a specific minimum alveolar concentration can be employed to ensure adequate depth of anesthesia. Maintenance of minimum alveolar concentrations above 0.7 has been shown to decrease the incidence of awareness. ${ }^{58}$ However, in cases in which a volatile anesthetic is not used (ie, total intravenous anesthesia), cases where anesthetic levels are minimized (trauma, cardiovascular anesthesia), or patients otherwise at risk for awareness, surrogate measures of anesthetic depth may help guide the anesthetist and prevent awareness. Use of the EEG has been the most popular surrogate to monitor depth of anesthesia. Typically, these monitors involve a specified algorithm to process raw EEG waveforms and supply a dimensionless number that indicates the patient's level of consciousness.

Proponents of the use of processed EEG for monitoring depth of anesthesia argue that these monitors allow precise titration of anesthetic levels, thereby reducing total amounts of anesthetic, reducing postanesthesia recovery time, and improving quality of recovery from a patient's perspective. ${ }^{59}$ Processed EEG-guided anesthesia has also been shown to reduce the incidence of intraoperative recall in patients at high risk for awareness. ${ }^{60}$ Other trials, however, failed to show benefit of processed EEG-guided anesthesia. In a large trial of 1,100 patients and over 3.3 million data points, processed EEG failed to correlate with end-tidal anesthetic concentration and was insensitive to changes in end-tidal anesthetic concentration. ${ }^{61}$ Another large-scale prospective study of over 6,000 subjects comparing processed EEG-guided protocols and end-tidal anesthetic concentration-guided protocols for maintenance of anesthesia found no difference in the amount of anesthesia used or the rate of awareness between the groups. ${ }^{62}$ Due to patient variability and insensitivity of processing algorithms, some authors have advocated the use of raw EEG waveforms to monitor depth of anesthesia. ${ }^{63}$

\section{Ultrasound}

Ultrasonography is an imaging technique utilizing an oscillating sound pressure wave at very high frequency. Its use allows the real-time imaging of structures such as bone, muscle, tendons, nerves, and blood vessels. It is relatively 
inexpensive compared with other imaging techniques, and due to its portability has become a ubiquitous tool for anesthesiologists. Besides the application of US in TEE, which has been described, the use of US for placement of central venous access and for the administration of peripheral nerve blockade is now commonplace.

Use of US for central venous cannulation improves the success rate and reduces the rate of complications. In a sentinel study comparing US-guided internal jugular venous catheterization with conventional, anatomic landmark-guided placement, use of US resulted in greater success and fewer needle passes per attempt. ${ }^{64}$ In addition, in cases where conventional methods resulted in failure, the use of US resulted in successful rescue cannulation. ${ }^{64}$ Similar studies found that US use resulted in fewer attempts and faster time to achieve success compared with conventional methods. ${ }^{65,66}$ A study by Karakitsos et $\mathrm{al}^{67}$ showed that US-guided internal jugular vein catheter insertion not only improved success and time to cannulation but also reduced the incidence of carotid artery puncture, hematoma, pneumothorax, and catheter-associated infections. A meta-analysis of 18 studies analyzing the efficacy of US in central venous cannulation corroborated the findings of these studies, showing that the use of US reduces complication rates and improves success rates. ${ }^{68}$ However, the author cautioned that the low rates of complications may not justify the cost of using this technology. ${ }^{68}$ In addition, the author concludes that clinicians trained in both approaches will not benefit from using US, and that training clinicians exclusively in using US will hinder their ability to place central venous lines when the technology is unavailable. ${ }^{68}$ Nevertheless, despite these concerns, the ASA task force on central venous access recommends the use of real-time US for placement of internal jugular vein catheters. ${ }^{69}$

US is used not only for placement of CVCs but also by anesthesiologists for guidance for peripheral nerve blockade. Over the last 5 years, US-guided regional anesthesia (UGRA) has become increasingly popular, displacing landmark-based techniques. Numerous studies have shown that UGRA is superior to conventional landmark-based techniques in reducing performance time for block placement, improving success rate, and reducing onset time of block..$^{70-72}$ In addition, because US allows for direct visualization of structures beneath the skin, its use has been associated with decreased vascular puncture and unintended paresthesias. ${ }^{70,71,73-75}$ Despite the fact that UGRA is still relatively new, there exists a large amount of scientific support for its supremacy in guiding anesthesiologists in regional block placement. It has been noted that use of US has changed clinical decision making by allowing regional anesthesia to be offered on a more widespread basis. ${ }^{76}$

\section{Target controlled infusion software and closed-loop drug delivery systems}

Opioid and sedative-hypnotic drugs are commonly given as infusions for maintenance of anesthesia and for conscious sedation. Drug infusion rates can be set and manually adjusted by the anesthesiologist, or they can be automatically adjusted by computerized software with imputed mathematical pharmacokinetic/pharmacodynamic models in what is known as target controlled infusion (TCI). In TCI, the anesthesiologist sets a target plasma or effect site concentration of the drug to be administered, and a computer automatically adjusts the infusion rate as predicted by the mathematical model. TCI is widespread internationally but is not US Food and Drug Administration (FDA) approved for clinical use in the United States. Proponents of TCI argue that TCI offers the anesthesiologist the ability to react to a concentration versus effect relationship that stays constant with time. ${ }^{77}$ In contrast, manually controlled drug delivery is based upon an infusion rate versus an effect relationship that constantly changes with time, making it difficult to characterize the drug concentration-effect relationship. ${ }^{77}$

Numerous studies have reported the benefits of TCI. In a study examining TCI delivery of remifentanil versus manually controlled delivery in spontaneously ventilating patients, Moerman et $\mathrm{al}^{78}$ found that TCI delivery resulted in less adjunct propofol administration and decreased incidence of apnea and hypopnea. TCI has been shown to result in a more rapid emergence from anesthesia and has been associated with a decreased incidence of postoperative nausea and vomiting and earlier discharge from the postanesthesia care unit. ${ }^{79,80}$ Gale et al $^{81}$ found no difference in depth of anesthesia when propofol was administered by TCI or manually. In addition, in a large analysis of 20 trials to assess the effectiveness of propofol TCI versus manually controlled propofol administration, Leslie et $\mathrm{al}^{82}$ found no clinically significant differences in anesthetic depth or adverse events. Use of TCI is associated with a higher cost compared with manually controlled anesthesia. ${ }^{80,82}$ It has been argued by some that because the incidence of adverse events directly related to drug administration and dosing is rare, studies demonstrating superiority of TCI over manually controlled administration will be lacking. ${ }^{83}$

With the development and international implementation of TCI systems, movement toward closed-loop drug 
delivery systems has intensified. A closed-loop system, by definition, is a system that automatically changes output based upon changes to an input signal. With drug delivery, closed-loop systems utilize TCI software, but the infusion rate is adjusted automatically in response to internal input such as physiologic variables or processed EEG values. Closed-loop drug delivery systems have been used experimentally for years. Studies examining the performance of closed-loop propofol administration for both induction and maintenance of anesthesia using processed EEG as an input variable have shown promising safety profiles and beneficial recovery times. ${ }^{84,85}$ Recently, closed-loop drug delivery has gained widespread attention with the introduction of a new propofol delivery device that has been FDA approved for use for conscious sedation for endoscopic procedures (Sedasys ${ }^{\circledR}$; Ethicon Endo-Surgery, Inc., Somerville, NJ, USA [United States patent fda.gov PO80009]). This device comprises two major components. The first is a bedside monitoring unit, which records vital signs (noninvasive blood pressure, ECG, pulse oximetry, and automated patient response). The second is a procedure room unit, which comprises the patient monitor, TCI software, and propofol infusion device. An oxygen delivery adapter is used for oxygen delivery and capnography. The clinician enters the patient weight and maintenance propofol infusion rate. The system calculates an initial loading dose based upon the recorded weight and imputed guidelines built in to the infusion software. The maintenance propofol infusion rate is started at the rate specified by the clinician. The rate is adjusted based upon input variables from the device that correlate to oversedation, including decreases in pulse oximetry and respiratory rate and/or reductions in patient response. Based upon the severity of these decreases, the device automatically stops and subsequently reduces the infusion rate or stops the infusion completely. Since the announcement of FDA approval of this device, the ASA has developed a strategic approach to understand computer-assisted patient sedation and ensure its safe and efficient introduction into clinical practice (Guidance for directors of anesthesia service for computer-assisted personalized sedation [CAPS] devices; http://www.asahq.org). With the recent FDA approval of this novel closed-loop device, evaluation of closed-loop drug delivery in a clinical setting will become feasible.

\section{Conclusion}

Without question, anesthesiologists are reliant on medical devices for their work. New devices and advances in older devices have changed the manner in which anesthetics are administered. Recent developments in technology, including fiberoptic technology and advances in computational processing and mathematical modeling, and also in physiologic monitoring have not only advanced the field of anesthesiology but made its practice more efficient. More importantly, new developments in anesthetic devices have made anesthesia safer for each and every patient exposed to its science.

\section{Disclosure}

The authors report no conflicts of interest in this work.

\section{References}

1. Mark JB. Central venous pressure monitoring: clinical insights beyond the numbers. J Cardiothorac Vasc Anesth. 1991;5:163-173.

2. Roy S, Couture P, Qizilbash B, et al. Hemodynamic pressure waveform analysis in predicting fluid responsiveness. J Cardiothorac Vasc Anesth. 2013;27:676-680

3. Forrester JS, Diamond G, McHugh TJ, Swan HJ. Filling pressures in the right and left sides of the heart in acute myocardial infarction. A reappraisal of central-venous-pressure monitoring. $N$ Engl J Med. 1971;285:190-193.

4. Murphy GS, Nitsun M, Vender JS. Is the pulmonary artery catheter useful? Best Pract Res Clin Anaesthesiol. 2005;19:97-110.

5. Eisenberg PR, Jaffe AS, Schuster DP. Clinical evaluation compared to pulmonary artery catheterization in the hemodynamic assessment of critically ill patients. Crit Care Med. 1984;12:549-553.

6. Quinn K, Quebbeman EJ. Pulmonary artery pressure monitoring in the surgical intensive care unit. Benefits vs difficulties. Arch Surg. 1981;116:872-876.

7. Tuchschmidt J, Sharma OP. Impact of hemodynamic monitoring in a medical intensive care unit. Crit Care Med. 1987;15:840-843.

8. Mimoz O, Rauss A, Rekik N, Brun-Buisson C, Lemaire F, Brochard L. Pulmonary artery catheterization in critically ill patients: a prospective analysis of outcome changes associated with catheter-prompted changes in therapy. Crit Care Med. 1994;22:573-579.

9. Staudinger T, Locker GJ, Laczika K, et al. Diagnostic validity of pulmonary artery catheterization for residents at an intensive care unit. J Trauma. 1998;44:902-906.

10. Wilson J, Woods I, Fawcett J, Whall R, Dibb W, Morris C, McManus E. Reducing the risk of major elective surgery: randomised controlled trial of preoperative optimisation of oxygen delivery. BMJ. 1999;318: 1099-1103.

11. Bishop MH, Shoemaker WC, Appel PL, et al. Prospective, randomized trial of survivor values of cardiac index, oxygen delivery, and oxygen consumption as resuscitation endpoints in severe trauma. $J$ Trauma. 1995;38:780-787.

12. Fleming A, Bishop M, Shoemaker W, et al. Prospective trial of supranormal values as goals of resuscitation in severe trauma. Arch Surg. 1992;127:1175-1179; discussion 1179-1181.

13. Connors AF Jr, Speroff T, Dawson NV, et al. The effectiveness of right heart catheterization in the initial care of critically ill patients. SUPPORT Investigators. JAMA. 1996;276:889-897.

14. Valentine RJ, Duke ML, Inman MH, et al. Effectiveness of pulmonary artery catheters in aortic surgery: a randomized trial. J Vasc Surg. 1998;27:203-211; discussion 211-212.

15. Stewart RD, Psyhojos T, Lahey SJ, Levitsky S, Campos CT. Central venous catheter use in low-risk coronary artery bypass grafting. Ann Thorac Surg. 1998;66:1306-1311.

16. Ramsey SD, Saint S, Sullivan SD, Dey L, Kelley K, Bowdle A. Clinical and economic effects of pulmonary artery catheterization in nonemergent coronary artery bypass graft surgery. J Cardiothorac Vasc Anesth. 2000;14:113-118. 
17. Practice guidelines for pulmonary artery catheterization. A report by the American Society of Anesthesiologists Task Force on Pulmonary Artery Catheterization. Anesthesiology. 1993;78:380-394.

18. American Society of Anesthesiologists Task Force on Pulmonary Artery Catheterization. Practice guidelines for pulmonary artery catheterization: an updated report by the American Society of Anesthesiologists Task Force on Pulmonary Artery Catheterization. Anesthesiology. 2003;99:988-1014.

19. Oxorn DC. Intraoperative echocardiography. Heart. 2008;94: 1236-1243.

20. Matsumoto M, Oka Y, Strom J, et al. Application of transesophageal echocardiography to continuous intraoperative monitoring of left ventricular performance. Am J Cardiol. 1980;46:95-105.

21. Bergquist BD, Bellows WH, Leung JM. Transesophageal echocardiography in myocardial revascularization: II. Influence on intraoperative decision making. Anesth Analg. 1996;82:1139-1145.

22. Mishra M, Chauhan R, Sharma KK, et al. Real-time intraoperative transesophageal echocardiography - how useful? Experience of 5,016 cases. J Cardiothorac Vasc Anesth. 1998;12:625-632.

23. Couture P, Denault AY, McKenty S, et al. Impact of routine use of intraoperative transesophageal echocardiography during cardiac surgery. Can J Anaesth. 2000;47:20-26.

24. Guzeltas A, Ozyilmaz I, Tanidir C, et al. The significance of transesophageal echocardiography in assessing congenital heart disease: our experience. Congenit Heart Dis. Epub September 19, 2013.

25. Bettex DA, Schmidlin D, Bernath MA, et al. Intraoperative transesophageal echocardiography in pediatric congenital cardiac surgery: a two-center observational study. Anesth Analg. 2003;97: 1275-1282

26. Stevenson JG. Role of intraoperative transesophageal echocardiography during repair of congenital cardiac defects. Acta Paediatr Suppl. 1995;410:23-33.

27. Ozier Y, Klinck JR. Anesthetic management of hepatic transplantation. Curr Opin Anaesthesiol. 2008;21:391-400.

28. Wax DB, Torres A, Scher C, Leibowitz AB. Transesophageal echocardiography utilization in high-volume liver transplantation centers in the United States. J Cardiothorac Vasc Anesth. 2008;22:811-813.

29. Wesseling KH, Purschke R, Smith NT, Wust HJ, de Wit B, Weber HA. A computer module for the continuous monitoring of cardiac output in the operating theatre and the ICU. Acta Anaesthesiol Belg. 1976; 27 Suppl:327-341.

30. Berkenstadt H, Margalit N, Hadani M, et al. Stroke volume variation as a predictor of fluid responsiveness in patients undergoing brain surgery. Anesth Analg. 2001;92:984-989.

31. Cannesson M, Slieker J, Desebbe O, et al. The ability of a novel algorithm for automatic estimation of the respiratory variations in arterial pulse pressure to monitor fluid responsiveness in the operating room. Anesth Analg. 2008;106:1195-1200, table of contents.

32. Nordstrom J, Hallsjo-Sander C, Shore R, Bjorne H. Stroke volume optimization in elective bowel surgery: a comparison between pulse power wave analysis (LiDCOrapid) and oesophageal Doppler (CardioQ). Br J Anaesth. 2013;110:374-380.

33. Davies SJ, Minhas S, Wilson RJ, Yates D, Howell SJ. Comparison of stroke volume and fluid responsiveness measurements in commonly used technologies for goal-directed therapy. J Clin Anesth. 2013;25: 466-474.

34. Yamada T, Tsutsui M, Sugo Y, et al. Multicenter study verifying a method of noninvasive continuous cardiac output measurement using pulse wave transit time: a comparison with intermittent bolus thermodilution cardiac output. Anesth Analg. 2012;115:82-87.

35. Manecke GR Jr, Auger WR. Cardiac output determination from the arterial pressure wave: clinical testing of a novel algorithm that does not require calibration. J Cardiothorac Vasc Anesth. 2007;21:3-7.

36. Murphy P. A fibre-optic endoscope used for nasal intubation. Anaesthesia. 1967;22:489-491.

37. Lee AC, Wu CL, Feins RH, Ward DS. The use of fiberoptic endoscopy in anesthesia. Chest Surg Clin N Am. 1996;6:329-347.
38. Ovassapian A. The flexible bronchoscope. A tool for anesthesiologists. Clin Chest Med. 2001;22:281-299.

39. American Society of Anesthesiologists Task Force on Management of the Difficult Airway. Practice guidelines for management of the difficult airway: an updated report by the American Society of Anesthesiologists Task Force on Management of the Difficult Airway. Anesthesiology. 2003;98:1269-1277.

40. Rogers SN, Benumof JL. New and easy techniques for fiberoptic endoscopy-aided tracheal intubation. Anesthesiology. 1983;59: 569-572.

41. Peterson GN, Domino KB, Caplan RA, Posner KL, Lee LA, Cheney FW. Management of the difficult airway: a closed claims analysis. Anesthesiology. 2005;103:33-39.

42. Rosenblatt WH, Wagner PJ, Ovassapian A, Kain ZN. Practice patterns in managing the difficult airway by anesthesiologists in the United States. Anesth Analg. 1998;87:153-157.

43. Benumof JL. Management of the difficult adult airway. With special emphasis on awake tracheal intubation. Anesthesiology. 1991;75: 1087-1110.

44. Howard-Quijano KJ, Huang YM, Matevosian R, Kaplan MB, Steadman RH. Video-assisted instruction improves the success rate for tracheal intubation by novices. Br J Anaesth. 2008;101:568-572.

45. Kaplan MB, Ward DS, Berci G. A new video laryngoscope-an aid to intubation and teaching. J Clin Anesth. 2002;14:620-626.

46. Maharaj CH, Costello JF, McDonnell JG, Harte BH, Laffey JG. The Airtraq as a rescue airway device following failed direct laryngoscopy: a case series. Anaesthesia. 2007;62:598-601.

47. Frova G. Do videolaryngoscopes have a new role in the SIAARTI difficult airway management algorithm? Minerva Anestesiol. 2010;76: 637-640.

48. Rosenstock CV, Thogersen B, Afshari A, Christensen AL, Eriksen C, Gatke MR. Awake fiberoptic or awake video laryngoscopic tracheal intubation in patients with anticipated difficult airway management: a randomized clinical trial. Anesthesiology. 2012;116:1210-1216.

49. Xue FS, Cheng Y, Li RP. Awake intubation with video laryngoscope and fiberoptic bronchoscope in difficult airway patients. Anesthesiology. 2013;118:462-463.

50. Brain AI. The laryngeal mask - a new concept in airway management. Br J Anaesth. 1983;55:801-805.

51. Yu SH, Beirne OR. Laryngeal mask airways have a lower risk of airway complications compared with endotracheal intubation: a systematic review. J Oral Maxillofac Surg. 2010;68:2359-2376.

52. Keller C, Brimacombe J, Bittersohl J, Lirk P, von Goedecke A. Aspiration and the laryngeal mask airway: three cases and a review of the literature. Br J Anaesth. 2004;93:579-582.

53. Daum RE, O'Reilly BJ. The laryngeal mask airway in ENT surgery. J Laryngol Otol. 1992;106:28-30.

54. Ebersole JS. EEG source modeling. The last word. J Clin Neurophysiol. 1999; 16:297-302.

55. Jordan KG. Emergency EEG and continuous EEG monitoring in acute ischemic stroke. J Clin Neurophysiol. 2004;21:341-352.

56. Deriu GP, Milite D, Mellone G, Cognolato D, Frigatti P, Grego F. Clamping ischemia, threshold ischemia and delayed insertion of the shunt during carotid endarterectomy with patch. J Cardiovasc Surg (Torino). 1999;40:249-255.

57. Krul JM, van Gijn J, Ackerstaff RG, Eikelboom BC, Theodorides T, Vermeulen FE. Site and pathogenesis of infarcts associated with carotid endarterectomy. Stroke. 1989;20:324-328.

58. Avidan MS, Zhang L, Burnside BA, et al. Anesthesia awareness and the bispectral index. N Engl J Med. 2008;358:1097-1108.

59. Recart A, Gasanova I, White PF, et al. The effect of cerebral monitoring on recovery after general anesthesia: a comparison of the auditory evoked potential and bispectral index devices with standard clinical practice. Anesth Analg. 2003;97:1667-1674.

60. Punjasawadwong Y, Boonjeungmonkol N, Phongchiewboon A. Bispectral index for improving anaesthetic delivery and postoperative recovery. Cochrane Database Syst Rev. 2007;4:CD003843. 
61. Whitlock EL, Villafranca AJ, Lin N, et al. Relationship between bispectral index values and volatile anesthetic concentrations during the maintenance phase of anesthesia in the B-Unaware trial. Anesthesiology. 2011;115:1209-1218.

62. Avidan MS, Jacobsohn E, Glick D, et al. Prevention of intraoperative awareness in a high-risk surgical population. N Engl J Med. 2011;365: 591-600.

63. Bennett C, Voss LJ, Barnard JP, Sleigh JW. Practical use of the raw electroencephalogram waveform during general anesthesia: the art and science. Anesth Analg. 2009;109:539-550.

64. Mallory DL, McGee WT, Shawker TH, et al. Ultrasound guidance improves the success rate of internal jugular vein cannulation. A prospective, randomized trial. Chest. 1990;98:157-160.

65. Koski EM, Suhonen M, Mattila MA. Ultrasound-facilitated central venous cannulation. Crit Care Med. 1992;20:424-426.

66. Slama M, Novara A, Safavian A, Ossart M, Safar M, Fagon JY. Improvement of internal jugular vein cannulation using an ultrasoundguided technique. Intensive Care Med. 1997;23:916-919.

67. Karakitsos D, Labropoulos N, De Groot E, et al. Real-time ultrasound-guided catheterisation of the internal jugular vein: a prospective comparison with the landmark technique in critical care patients. Crit Care. 2006;10:R162.

68. Keenan SP. Use of ultrasound to place central lines. J Crit Care. 2002;17:126-137.

69. American Society of Anesthesiologists Task Force on Central Venous Access, Rupp SM, Apfelbaum JL, Blitt C, et al. Practice guidelines for central venous access: a report by the American Society of Anesthesiologists Task Force on Central Venous Access. Anesthesiology. 2012;116:539-573.

70. Brull R, Lupu M, Perlas A, Chan VW, McCartney CJ. Compared with dual nerve stimulation, ultrasound guidance shortens the time for infraclavicular block performance. Can J Anaesth. 2009;56:812-818.

71. Chan VW, Perlas A, McCartney CJ, Brull R, Xu D, Abbas S. Ultrasound guidance improves success rate of axillary brachial plexus block. Can J Anaesth. 2007;54:176-182.

72. Danelli G, Fanelli A, Ghisi D, et al. Ultrasound vs nerve stimulation multiple injection technique for posterior popliteal sciatic nerve block. Anaesthesia. 2009;64:638-642.

73. Sauter AR, Dodgson MS, Stubhaug A, Halstensen AM, Klaastad O. Electrical nerve stimulation or ultrasound guidance for lateral sagittal infraclavicular blocks: a randomized, controlled, observer-blinded, comparative study. Anesth Analg. 2008;106:1910-1915.
74. Mariano ER, Loland VJ, Bellars RH, et al. Ultrasound guidance versus electrical stimulation for infraclavicular brachial plexus perineural catheter insertion. J Ultrasound Med. 2009;28:1211-1218.

75. Mariano ER, Loland VJ, Sandhu NS, et al. Ultrasound guidance versus electrical stimulation for femoral perineural catheter insertion. J Ultrasound Med. 2009;28:1453-1460.

76. Antonakakis JG, Ting PH, Sites B. Ultrasound-guided regional anesthesia for peripheral nerve blocks: an evidence-based outcome review. Anesthesiol Clin. 2011;29:179-191.

77. Egan TD, Shafer SL. Target-controlled infusions for intravenous anesthetics: surfing USA not! Anesthesiology. 2003;99:1039-1041.

78. Moerman AT, Herregods LL, De Vos MM, Mortier EP, Struys MM. Manual versus target-controlled infusion remifentanil administration in spontaneously breathing patients. Anesth Analg. 2009;108:828-834.

79. Muller T, Ludwig A, Biro P. Two distinct application habits for propofol: an observational study. Eur J Anaesthesiol. 2010;27:265-269.

80. Suttner S, Boldt J, Schmidt C, Piper S, Kumle B. Cost analysis of target-controlled infusion-based anesthesia compared with standard anesthesia regimens. Anesth Analg. 1999;88:77-82.

81. Gale T, Leslie K, Kluger M. Propofol anaesthesia via target controlled infusion or manually controlled infusion: effects on the bispectral index as a measure of anaesthetic depth. Anaesth Intensive Care. 2001;29: 579-584.

82. Leslie K, Clavisi O, Hargrove J. Target-controlled infusion versus manually-controlled infusion of propofol for general anaesthesia or sedation in adults. Cochrane Database Syst Rev. 2008;3:CD006059.

83. Bazaral MG, Ciarkowski A. Food and Drug Administration regulations and computer-controlled infusion pumps. Int Anesthesiol Clin. 1995;33: 45-63.

84. De Smet T, Struys MM, Neckebroek MM, Van den Hauwe K, Bonte S, Mortier EP. The accuracy and clinical feasibility of a new bayesianbased closed-loop control system for propofol administration using the bispectral index as a controlled variable. Anesth Analg. 2008;107: 1200-1210.

85. Struys MM, De Smet T, Versichelen LF, Van De Velde S, Van den Broecke R, Mortier EP. Comparison of closed-loop controlled administration of propofol using Bispectral Index as the controlled variable versus "standard practice" controlled administration. Anesthesiology. 2001;95:6-17.
Medical Devices: Evidence and Research

\section{Publish your work in this journal}

Medical Devices: Evidence and Research is an international, peerreviewed, open access journal that focuses on the evidence, technology, research, and expert opinion supporting the use and application of medical devices in the diagnosis, treatment and management of clinical conditions and physiological processes. The identification of novel

\section{Dovepress}

devices and optimal use of existing devices which will lead to improved clinical outcomes and more effective patient management and safety is a key feature. The manuscript management system is completely online and includes a quick and fair peer-review system. Visit http://www. dovepress.com/testimonials.php to read real quotes from authors. 